Shareholder of: AstraZeneca, Employee of: Medlmmune, G. Illei Shareholder of: AstraZeneca, Employee of: Medlmmune LLC, K. Ranade Employee of: Medlmmune LLC

DOI: 10.1136/annrheumdis-2017-eular.3719

\section{OP0302 SIGNIFICANT REDUCTIONS OF PATHOGENIC AUTOANTIBODIES BY SYNERGETIC RITUXIMAB AND BELIMUMAB TREATMENT EFFECTIVELY INHIBITS NEUTROPHIL EXTRACELLULAR TRAPS IN SEVERE, REFRACTORY SLE - THE SYNBIOSE STUDY}

T. Kraaii ${ }^{1}$, S.W. Kamerling ${ }^{1}$, J.A. Bakker ${ }^{2}$, T.W. Huizinga ${ }^{3}$, T.J. Rabelink ${ }^{1}$, C. van Kooten ${ }^{1}$, Y.K. Teng ${ }^{1} .{ }^{1}$ Nephrology; ${ }^{2}$ Clinical Chemistry; ${ }^{3}$ Rheumatology, LUMC, Leiden, Netherlands

Background: Neutrophil extracellular traps (NETs) are extracellular, decondensed DNA strands covered with antimicrobial proteins that are part of the first-line defence against pathogens. However, in SLE, overall release of NETs is increased and degradation of NETs is impaired leading to a high amount of extracellular nuclear material, potentially leading to formation of SLE-specific antibodies. These pathogenic autoantibodies deposit in glomeruli in lupus nephritis (LN) and perpetuate autoimmunity by inducing more NETs. The present study hypothesized that combining anti-CD20 mediated B-cell depletion with BAFF (B-cell activating factor) inhibition can target autoreactive plasma cells and thereby effectively reduce pathogenic autoantibodies and NET induction in severe SLE.

Objectives: The present study aimed to investigate whether Rituximab (RTX) + Belimumab (BLM) affected pathogenic antibodies in relation to NET induction in severe refractory SLE.

Methods: As part of a phase 2 proof-of-concept study, the SynBioSe study, serum levels of anti-DNA autoantibodies were measured in severe, refractory SLE patients before and after treatment with RTX following BLM. Additionally, ex vivo NET induction was assessed before and after treatment with a novel highly sensitive method based on 3D confocal laser scanning microscopy. In this assay, healthy neutrophils are incubated with $10 \%$ serum of patients and healthy controls. Furthermore, we investigated whether NET induction was mediated by immune complexes.

Results: The study included 10 severe, refractory SLE patients with lupus nephritis and 1 patient with neuropsychiatric lupus. NET induction was found to be high at baseline with a median fold induction of 4.5 [range 2.6-11.7]. After 24 weeks, NET induction was significantly decreased (median fold NET induction of 1.6 [0.4-6.1], $\mathrm{p}=0.01$ ). In addition, treatment with $\mathrm{RTX}+\mathrm{BLM}$ led to significant reduction of anti-dsDNA antibodies at week 24 with a median of $35 \mathrm{IU} / \mathrm{ml}$ [range $10-374]$ compared to 120 [18-505] at baseline $(p=0.012)$. Total immunoglobulin levels temporarily declined but returned to screening levels at week 24. NET induction correlated significantly with anti-dsDNA antibody levels $(r=0.42, p=0.03)$ and with SLEDAI scores $(r=0.53, p=0.003)$. Therefore, we examined whether the observed NET induction could be explained by circulating immune complexes (ICx). ICx were degraded by pre-incubating anti-dsDNA positive SLE sera with nuclease, resulting in a significant decrease in NET induction (median \% decrease of 91.7 [range 67.6-98.1]). In addition, depletion of IgG from anti-dsDNA positive SLE sera resulted in significantly lower NET induction. Finally, immobilized IgG isolated from anti-dsDNA positive SLE sera, but not of control serum, resulted in significant NET induction.

Conclusions: Within refractory SLE patients, RTX + BLM resulted in concordant reductions in pathogenic anti-dsDNA antibodies and NET-inducing capacity. This study strongly suggests that NET induction in SLE is mediated by immune complexes, providing a possible explanation underpinning the clinical benefits of RTX+BLM in SLE. Trial registration:ClinicalTrials.gov NCT02284984

Disclosure of Interest: None declared

DOI: 10.1136/annrheumdis-2017-eular.5706

\section{OP0303 THE SALIVARY GLAND SECRETOME AS A POTENTIAL NEW TOOL TO IDENTIFY BIOMARKERS OF DRYNESS AND IMMUNOPATHOLOGY IN PRIMARY SJÖGREN'S SYNDROME AND NON-AUTOIMMUNE SICCA PATIENTS}

S.L. Blokland ${ }^{1,2}$, M.R. Hillen ${ }^{1,2}$, A.A. Kruize ${ }^{2}$, W. de Jager ${ }^{1}$, A. Pandit ${ }^{1}$, J.A. van Roon ${ }^{1,2}$, T.R. Radstake ${ }^{1,2} .{ }^{1}$ Laboratory of Translational Immunology;

${ }^{2}$ Rheumatology \& Clinical Immunology, UMC Utrecht, Utrecht, Netherlands

Background: Salivary gland biopsy is essential in primary Sjögren's syndrome (pSS) diagnostics. However, tissue analysis using traditional methodology has several limitations including inaccurate quantification of lymphocytic infiltration and poor correlation with dryness. To perform biomarker identification in the target organ, tissue would have to be sacrificed. By performing saliva proteomics the biopsy tissue can be saved, but hitherto, this technique has not yielded consistent biomarkers and is limited by the absence of saliva production by many sicca patients.

Objectives: We aimed to explore whether Luminex analysis of a broad panel of cytokines in salivary gland biopsy supernatants (secretome) could provide biomarkers to stratify sicca patients and could give insights into pathogenesis.

Methods: Labial salivary gland (LSG) tissues were rinsed after biopsy and incubated in $200 \mu \mathrm{L}$ of saline for $1 \mathrm{~h}$ at room temperature. Tissue supernatants were rendered cell-free, frozen in liquid nitrogen and stored at $-80^{\circ} \mathrm{C}$. In supernatants from pSS and non-Sjögren's sicca (nSS) patients 104 targets were measured by Luminex. Eight pSS and $8 \mathrm{nSS}$ patients were selected for analysis based on matched biopsy weights. Results from this discovery cohort were validated in an additional cohort ( $n=18 \mathrm{nSS}, \mathrm{n}=16$ incomplete SS: iSS, $n=26 \mathrm{pSS}$ ) and correlations with clinical parameters were assessed. Non-SS were defined as sicca patients without lymphocytic infiltration in the salivary gland biopsy or anti-SSA/SSB autoantibodies. Incomplete SS patients were defined as sicca patients having lymphocytic infiltration (lymphocytic focus score (LFS) $>0$ ) and/or anti-SSA/SSB autoantibodies but do not fulfill the AECG classification criteria and are not diagnosed as pSS.

Results: Levels of 20 cytokines were significantly different between the nSS and pSS patients in the discovery cohort $(p \leq 0.05)$. These 20 and 13 additionally selected cytokines based on a trend towards statistical significance and/or literature, were measured in a validation cohort. Weights of the biopsies did not significantly differ: $59.8 \pm 48.1 \mathrm{mg}$ in nSS vs $72.7 \pm 45.2 \mathrm{mg}$ in iSS vs $67.4 \pm 28.6 \mathrm{mg}$ in pSS. Fifteen out of these 20 cytokines were validated. From the 13 cytokines 7 were significantly elevated in pSS vs nSS. In iSS CXCL10 (IP-10) and CCL19 (MIP-3 $\beta$ ) were significantly elevated. Cytokines correlating with LFS, ESSDAI, ESSPRI, \% IgG and IgM+ plasma cells in LSG, Schirmer and/or serum IgG with Spearman $r>0.4$ and $p<0.05$ in $p S S$ were selected for classification tree analysis, these were IL-2, IL-3, IFN- $\beta$, IL-21, CXCL13 (BLC), CXCL10 and CCL19. Using CXCL13 and IL-21 levels, $87.5 \%$ of pSS patients could be classified correctly. Based on the used cut off levels, 5 nSS and 9 iSs patients would be classified as pSS. Follow up of these patients may reveal development of pSS.

Conclusions: Elevated levels of numerous cytokines were found in LSG biopsy secretomes from pSS patients versus non-autoimmune sicca patients correlating with clinical parameters. This method represents a novel tool to provide insights in pSS immunopathology and to identify therapeutic targets and biomarkers for diagnosis, prognosis and treatment response.

Disclosure of Interest: None declared

DOI: 10.1136/annrheumdis-2017-eular.1608

\section{OP0304 SELECTIVE INHIBITORS OF NUCLEAR EXPORT PREVENT LUPUS PROGRESSION BY TARGETING GERMINAL CENTER FORMATION AND AUTOREACTIVE ANTIBODY SECRETING CELLS}

J. Rangel-Moreno ${ }^{1}$, S. Cochran ${ }^{2}$, S. Tamir ${ }^{2}$, M. Lee ${ }^{2}$, S. Shacham ${ }^{2}$, J. Anolik ${ }^{1}$ ${ }^{1}$ Department of Medicine, Division of Allergy, Immunology and Rheumatology, University of Rochester Medical Center, Rochester; ${ }^{2}$ Karyopharm Therapeutics, Newton, United States

Background: Systemic lupus erythematosus (SLE) is a complex autoimmune disease characterized by simultaneous activation of the innate and adaptive arms of the immune system. The progression of the disease is unpredictable, making its treatment a challenge. Recently the nuclear export protein Exportin 1 (XPO1, also known as CRM1) has surfaced as an attractive target for the treatment of SLE and other inflammatory disorders. Selective Inhibitor of Nuclear Export (SINE) compounds are potent, orally available and well-tolerated XPO1 inhibitors. SINE compounds exert apoptotic and anti-inflammatory effects by mediating nuclear retention of important XPO1 cargos like the $\mathrm{NF}$ KB pathway regulatory protein, IкB

Objectives: Based on the central role of $\mathrm{NF}_{K \mathrm{~B}}$ signaling in the activation of immune cells in SLE, we decided to evaluate the therapeutic ability of SINE compounds to modulate experimental lupus progression.

Methods: To evaluate the efficacy of SINE compounds in a preclinical model of SLE, cohorts of lupus-prone mice with established disease (elevated anti-dsDNA antibody titer and proteinuria) were dosed with SINE compound or vehicle. We used flow cytometry to enumerate immune cells and immunofluorescence to visualize germinal centers $(\mathrm{GC})$ in spleen. Quantitative PCR was used to measure changes in mRNA expression for molecules key in plasma cell attraction and survival, and histology was used to evaluate inflammation, antibody deposition and pathology in kidneys of lupus-prone mice.

Results: We found that treatment with SINE compounds significantly prevented increases in proteinuria (proteinuria scores: Control: 2.12 \pm 1.12 ; SINE $(5 \mathrm{mg} / \mathrm{kg}$ ): $1.06 \pm 0.49 ; \operatorname{SINE}(7.5 \mathrm{mg} / \mathrm{kg}): 0.85 \pm 0.55)$ and drastically decreased IgG deposition and kidney pathology (glomerulonephritis, tubule damage and perivascular cuffing). Prevention of kidney damage was associated with a remarkable disruption of splenic GC, a significant reduction in the number of auto-reactive antibody secreting cells (ASC), and a decrease in the accumulation of auto-reactive ASC in the inflamed kidney. Reduced numbers of plasma cells in the inflamed kidney are likely due to the drastic decrease in the expression of molecules critical for PC attraction (CCL2, CXCL9, CXCL10, CXCL11) and survival (BAFF, APRIL). The potent effect of SINE compounds on GC and auto-reactive ASC is noticeable as early as 1 week after starting therapy. However, kinetics studies showed that a more pronounced elimination of GC and auto-reactive ASC is achieved after 8 weeks. Although SINE therapy has a drastic impact on spleen architecture, recovery experiments showed that complete recovery of immune cells in spleen occurred by 4 weeks. The reversible impact of SINE compounds on SLE provides a potential window of time for immunization of lupus patients.

Conclusions: SINE compounds have demonstrated efficacy in a murine model of SLE by reducing generation, survival and function of auto-reactive immune 